vanishing sperm. Researchers need to exercise great care in how they collect data and describe their methods and results in journals. This is not a call for a return to simple empiricism, in which data are seen as being supreme. We would do well to remember that the word "datum" means "given" and provides us with information based on what we choose to collect and what we choose to leave out. There is inherent bias in how we define the problem in the first place. Once measurement begins, bias and error are fundamental.

The misapplication of increasingly sophisticated statistical tests (of which regression is one of the simpler) is becoming commonplace. More rigorous application of legitimate methods of analysis is required, particularly when time series are being analysed. When inferences are being drawn over time we deserve more than simple analysis: we need to follow McKeown's example and seek corroborating evidence from a wide range of disciplines. ${ }^{6}$ Why not refer to the extensive data from veterinary research?

By the nature of their work epidemiologists erect hypotheses and invite others to test them to destruction. They run a constant occupational risk, that of being mistaken. Editors have their part to play in protecting this small occupational group from doing themselves damage. Alternatively, editors could consider printing the names of referees alongside articles. This would do nothing to allay anxieties, but it would certainly spread the blame.

STEPHEN FARROW Professor

Health Research Centre,

Middlesex University,

Enfield EN3 4SF

1 Carlsen E, Giwercman A, Keiding N, Skakkebaek NE. Evidence for the decreasing quality of semen during the past 50 years. $B M F$ 1992;305:609-12.

Bromwich P, Cohen J, Stewart I, Walker A. Decline in sperm counts: an artefact of changed reference range of normal? BMF 1994;309:19-22.

reference range of normal? $B M 7194,39: 19-22$.
Nelson CMK, Bunge RG. Semen analysis: evidence for changing parameters of male fertility potential. Fertil Steril 1974;25:503-7.

Macleod J, Wang Y. Male fertility potential in terms of semen quality: a review of the past, a study of the present. Fertil Steril 1979;31:103-16.

5 Polakoski KL, Zahler WL, Paulson JD. Demonstration of proacrosin and quantitation of acrosin in ejaculated human spermatozoa. Fertil Steril 1977;28:668-70.

6 McKeown T. The origins of human disease. Oxford: Basil Blackwell, 1988.

\title{
Triglyceride concentration and coronary heart disease
}

\section{Not yet proved of value as a screening test}

As a cardiac risk factor triglycerides have occupied an uneasy position for a long time: perpetually under a cloud of suspicion but never proved to cause or even have a definite association with the risk of coronary heart disease. There are good grounds for suspicion-biological plausibility and epidemiological data suggest that triglycerides might promote coronary heart disease and other forms of cardiovascular disease-but the epidemiological and clinical evidence is inconsistent and often flawed. Even recent recommendations calling for the use of triglyceride concentration in screening are qualified by two admissions: that the evidence for a causal link remains frail and that trials have not established any clinical benefit from measuring triglyceride concentration. ${ }^{1-3}$

Why should a final judgment about the importance of triglycerides in cardiovascular disease remain so elusive? One cause is the variability of measurements of triglyceride concentrations-much greater than for other common lipids. This is partly due to the laboratory and partly to "true" short term fluctuations. This variability helps explain why statistical techniques underestimate the association between triglyceride concentration and the risk of coronary heart disease. ${ }^{45}$ Clinicians can reduce the influence of physiological variability in triglyceride values by averaging repeated measurementsjust as they do when measuring cholesterol concentration or blood pressure. But blood for measurement of triglyceride concentrations should be drawn after a 12 hour fast, making repeated measurement inconvenient and unappealing.

Perhaps the greatest perplexity about the importance of triglycerides derives from their relation to high density lipoprotein cholesterol concentration. This has a strong inverse correlation with triglyceride concentration, and studies that include measurement of high density lipoprotein cholesterol usually find no independent association between triglycerides and coronary heart disease. ${ }^{6}$ Nevertheless, some recent studies have still failed to control for confounding by high density lipoprotein cholesterol. For example, the study by Lindenstrom and colleagues in this issue of the $B M F$ suggests an association between serum triglyceride concentration $^{7}$ and stroke ( $p$ 11); two other recent studies have reported, once again, that triglycerides and coronary heart disease are related. ${ }^{8}$ Yet all three investigations failed to adjust for high density lipoprotein cholesterol concentration, thereby contributing little to solving the question of whether triglycerides have additional predictive power beyond that of high density lipoprotein cholesterol.

Yet even if triglycerides do not have an independent association with coronary heart disease in multivariate analyses, might they be used to refine the estimation of coronary risk in some people? The Helsinki heart study found an interaction between triglycerides and cholesterol: most of the risk of coronary heart disease and nearly all the benefits of gemfibrozil treatment were confined to people with high concentrations of both triglyceride and cholesterol (particularly those with a ratio of low to high density lipoprotein of more than 5). ${ }^{10}$ Similar findings emerged from the prospective cardiovascular Munster study (PROCAM), an observational study. ${ }^{3}$ These results suggest that if triglyceride concentration is included with other lipid measurements, people might be identified who would benefit most from treatment. But the gaps and contradictions in published research make it clear that any such conclusion is speculative. Previous attempts to identify interactions among the lipid subclasses have had mixed results, either failing to detect important interactions or even finding inconsistent relations. For example, some studies found that triglycerides were associated with coronary heart disease only in subjects with low concentrations of cholesterol. ${ }^{112}$

The cholesterol-triglyceride interaction in the Helsinki heart study, while intriguing, was discovered incidentally and was not statistically significant. ${ }^{13}$ Even if the finding is valid its implications for treatment are uncertain. Does the interaction imply that patients with raised cholesterol and triglyceride concentrations should be given more aggressive treatment to lower cholesterol concentration, or does it imply that they should be treated specifically with a drug (such as gemfibrozil) that also lowers triglyceride concentration? Confirmation in other clinical trials will be needed before we learn whether and how measurement of triglyceride values is useful in guiding lipid lowering treatment in patients with raised cholesterol concentrations. 
Some reports have suggested that triglyceride concentration may be a more important risk factor in women than men, but this seems to be true only in univariate analyses. Most studies that adjust for high density lipoprotein cholesterol and other lipids do not support an independent association between serum triglyceride concentrations and coronary heart disease in women. ${ }^{611}$

What, then, can one fairly conclude? Has measurement of triglyceride concentrations a role in preventing cardiovascular disease? Despite the numerous studies, the use of triglyceride concentration in screening or in guiding lipid lowering treatment must still be seen as experimental. Precise measurements will be needed if triglyceride concentration is to be used to help identify people at high risk of coronary heart disease, but any such use seems limited-because of the greater convenience and predictive accuracy of measurements of high density lipoprotein cholesterol. Possibly measurements of triglyceride will identify people who would benefit from aggressive cholesterol lowering treatment or point to the best class of drugs for treating patients with hypercholesterolaemia. Both of these uses for measurement of triglycerides are speculative, however, and in both cases the test needs to be done only in someone found to have a raised cholesterol concentration.

As far as screening is concerned, measuring triglycerides is less convenient than measuring high density lipoprotein cholesterol or total cholesterol, and current evidence suggests that its value in risk classification would at best be limited. Even if the test gave additional information about risk it would not necessarily lead to better treatment decisions. Despite the many clinical trials of reducing raised concentrations of cholesterol, the application of total and low density lipoprotein cholesterol to treatment decisions remains controversial. ${ }^{14}$ There is far less evidence supporting the use of triglyceride concentrations.

Concern about resources for health care has raised the standards of evidence required for any general screening strategy. The useful screening test-as is well known-has to be safe, convenient, inexpensive, and to discriminate between those who do and do not benefit from further diagnostic evaluation or treatment. Further evidence may someday enable triglyceride concentration to meet these standards, but not yet.

ALAN M GARBER

Associate professor of medicine

Department of Veterans Affairs Medical Center,

Palo Alto, CA 94303,

USA

University of California, San Francisco, San Francisco, CA 94105,

USA

1 NIH Consensus Development Panel on Triglyceride, High-Density Lipoprotein, and Coronary Heart disease. NIH consensus conference: triglyceride, high-density lipoprotein, and coronary Heart Disease. FAMA 1993;269:505-10.

2 Hypertriglyceridaemia and vascular risk. Report of a meeting of physicians and scientists, University College London Medical School. Lancet 1993;342:781-7.

3 Assmann G, Schulte H. Relation of high-density lipoprotein cholesterol and triglycerides to incidence of atherosclerotic coronary artery disease (the PROCAM experience). Prospective incidence of atherosclerotic coronary artery disease (the

4 Phillips AN, Smith GD. How independent are "independent" effects? Relative risk estimation when correlated exposures are measured imprecisely. F Clin Epidemiol 1991;44:1223-31.

5 Davey Smith G, Song F, Sheldon TA. Cholesterol lowering and mortality: the importance of considering initial level of risk. $B M \mathcal{F}$ 1993;306:1367-73.

6 Avins AL, Haber RJ, Hulley SB. The status of hypertriglyceridemia as a risk factor for coronary heart disease. Clin Lab Med 1989;9:153-68.

7 Lindenstrom E, Boysen G, Nyboe J. Influence of total cholesterol, high density lipoprotein cholesterol, and triglycerides on risk of cerebrovascular disease. The Copenhagen city hear study. $B M F$ 1994;309:11-5.

8 Bengtsson C, Bjorkelund C, Lapidus L, Lissner L. Associations of serum lipid concentrations and obesity with mortality in women: 20 year follow up of participants in prospective population study in Gothenburg, Sweden. BMF 1993;307:1385-8.

9 Stensvold I, Tverdal A, Urdal P, Graff IS. Non-fasting serum triglyceride concentration and mortality from coronary heart disease and any cause in middle aged Norwegian women. $B M f$ 1993:307:1318-22.

10 Manninen V, Tenkanen L, Koskinen P. Joint effects of serum triglyceride and LDL cholestero and HDL cholesterol concentrations on coronary heart disease risk in the Helsinki heart study. Implications for treatment. Circulation 1992;85:37-45.

1. Austin MA. Plasma triglyceride and coronary heart disease. Arterioscler Thromb 1991;11:2-14.

12 Criqui MH, Heiss G, Cohn R, Cowan LD, Suchindran CM, Bangdiwala S, et al. Plasma triglyceride level and mortality from coronary heart disease. N Engl F Med 1993;328:1220-5.

13 Avins AL, Hulley SB. Joint effects of serum triglycerides and LDL and HDL cholesterol. Circulation 1993;87:300-1.

14 Hulley SB, Newman TB, Grady D, Garber AM, Baron RB, Browner WS. Should we be measuring blood cholesterol levels in young adults? FAMA 1993;269:1416-9.

\title{
Reducing the use of benzodiazepines in general practice
}

\author{
Explain the likely duration of treatment (short) before prescribing the first dose
}

Benzodiazepines are the most commonly used psychotropic drugs. ${ }^{12}$ When introduced they were embraced with enthusiasm by the medical profession and public alike, although since then a backlash has developed against them, which has culminated in legal actions against their manufacturers. The use of benzodiazepines has fallen steadily since the $1970 \mathrm{~s}^{3}$ Adverse effects have been highlighted, including the chance of a discontinuation syndrome after patients stop taking the drugs. ${ }^{4}$

Doctors have been exhorted not to prescribe these drugs and to try to stop their long term use. ${ }^{5}$ The exhortations have worked: a community survey in this week's journal does not confirm earlier popular notions of long term use of benzodiazepines ( $p$ 27)..$^{6}$ Benzodiazepines are now often regarded as suitable only for short term use, if they have a use at all. (This ignores their appropriate longer term use by some patients. ${ }^{7}$ ) The development of newer anxiolytics and hypnotics and roles for some newer antidepressants as anxiolytics may further alter patterns of use of benzodiazepines.

Educating the public about the risks and benefits of these drugs is the key to reducing their use. Educational programmes can be supplemented by written material and counselling during medical consultations. An educational programme directed at prescribers, people providing care, and patients (who also received training in relaxation) halved long term use of benzodiazepines among elderly residents in care. ${ }^{8}$ A study of 200 long term users found that more than half were using benzodiazepines as hypnotics and had done so for a median of 15 years. Use fell by almost a third after a simple mailing encouraged patients slowly to reduce then stop their drugs. ${ }^{3}$ Some of the patients may have been receiving the wrong treatment-insomnia may be a symptom of depression, which is better treated with antidepressants than benzodiazepines.

The appropriate use of benzodiazepines depends on a careful diagnosis and clear instructions regarding the drugs' use. ${ }^{9}$ For many patients general health advice; supportive counselling; and information on sleep, relaxation, and behavioural strategies, including cognitive behavioural therapy may suffice. These techniques can be learnt, and every practitioner should be able to provide them. They do not necessarily require long consultations and may be used over several brief consultations. Active participation in treatment reinforces the patients' responsibility for their health. Patients 